\title{
Hustensaft erhöht das Anaphylaxierisiko bei der Narkose
}

\section{In Norwegen reagierten Patienten während der Narkose gehäuft mit einer anaphylaktischen Reaktion. Als mögliche indirekte Ursache gilt das Antitussivum Pholcodin, das inzwischen vom Markt genommen wurde. Der Effekt dieser Maßnahme wurde jetzt beleuchtet.}

A naphylaxien nach Gabe von Muskelralaxanzien wie Suxamethonium bei Anästhesien nahmen in den letzten Jahrzehnten vor allem in Norwegen auf-

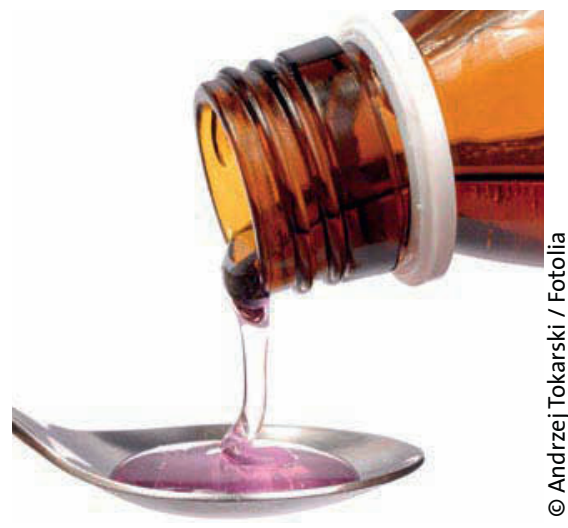

Das Antitussivum Pholcodin und das Muskelrelaxans Suxamethonium teilen sich eine quartäre Ammoniumgruppe. fällig zu. Dort lagen die Fallzahlen gar um ein Zehnfaches höher als bei den schwedischen Nachbarn. Der Hauptunterschied zwischen den beiden Ländern bestand in der Verwendung eines Hustensafts mit dem Wirkstoff Pholcodin $\left(\right.$ Tuxi $\left.^{\circledR}\right)$. Mit Suxamethonium hat er eine quartäre Ammoniumgruppe gemein, die für Kreuzreaktionen verantwortlich sein könnte. Tuxi ${ }^{\circledR}$ wurde im März 2007 vom norwegischen Markt genommen. Der Effekt dieser Maßnahme wurde an 300 Serumproben von als allergisch eingestuften Patienten untersucht, die auf ein Jahr vor bis drei Jahre nach diesem Zeitpunkt datierten.

Sensibilisierungen gegen Pholcodin, Suxamethonium und das ebenfalls ammoniumhaltige Morphin galten als bestätigt bei spezifischen-IgE-Konzentrationen von mindestens $0,35 \mathrm{kUA} / \mathrm{l}$.
Vor der Rücknahme von Tuxi ${ }^{\circledR}$ zeigten sich Sensibilisierungsraten von $11,0 \%$ gegen Pholcodin, 10,0\% gegen Morphin und 3,7\% gegen Suxamethonium. Im ersten Jahr danach waren nur noch 5,0\% gegen Pholcodin und 0,7\% gegen Suxamethonium sensibilisiert - zu Morphin lagen keine auswertbaren Daten vor. Zwei Jahre später betrugen die Sensibilisierungsraten $5,7 \%$ bei Pholcodin, 2,7\% bei Morphin und 0,3\% bei Suxamethonium. Im dritten Jahr waren Sensibilisierungen noch in 2,7, 1,3 bzw. $0,3 \%$ der Fälle nachweisbar. Nach diesen drei Jahren wurde auch eine signifikante Abnahme von Anaphylaxien unter Narkose verzeichnet.

Fazit: Die Marktrücknahme von Pholcodin führte nach dieser Studie zu einer signifikanten Abnahme von Sensibilisierungen gegen Pholcodin, Morphin und Suxamethonium. Gleichfalls sank innerhalb weniger Jahre die Zahl der Anaphylaxien unter Narkose. Andreas Fischer

Florwaag $\mathrm{E}$ et al. IgE-sensitization to the cough suppressant pholcodine and the effects of its withdrawal from the Norwegian market. Allergy 2011 Jan 17 [Epub ahead of print]

\section{Mukoviszidose: veränderte Immunantwort auf Antibiotika}

\section{Vielen Patienten mit zystischer Fibrose reagieren allergisch auf Anti- biotika, einen Eckpfeiler ihrer Behandlung. Die daran beteiligten Pathomechanismen standen im Fokus einer In-vitro-Studie.}

D as Centre for Drug Safety Science in Liverpool untersuchte im Rahmen einer internationalen Kooperation Immunantworten von T-Zell-Klonen, die von drei Patienten mit sowie von drei Patienten ohne zystische Fibrose gewonnen worden waren. Alle Studienteilnehmer waren gegen das Antibiotikum Sulfamethoxazol sensibiliert.

Bei den Patienten ohne zystische Fibrose konnten drei verschiedene T-ZellTypen beobachtet werden: Klone, die auf Sulfamethoxazol reagierten, Klone mit Reaktion auf Sulfamethoxazol-Stickstoffmonoxid sowie kreuzreagierende T-
Zell-Klone. Bei den Mukoviszidose-Patienten konnten nur auf SulfamethoxazolMonoxid reagierende und kreuzreagierende T-Zell-Klone identifiziert werden - jedoch keine Klone, die allein durch Sulfamethoxazol stimulierbar waren.

Bei den antigenpräsentierenden Zellen von Patienten ohne zystische Fibrose konnten zwei Varianten der Immunantwort nach Exposition mit Sulfamethoxazol nachgewiesen werden. Der erste Weg bestand in der direkten Interaktion mit dem Antibiotikum und TZellen. Ein zweiter Weg war die Metabolisierung von Sulfomethoxazol durch die antigenpräsentierenden Zellen mit Bindung der Abbauprodukte an zelluläre Proteine. Diese Addukte wiesen antigene Eigenschaften auf. Zellen von Patienten mit Mukoviszidose dagegen reagierten ausschließlich nur über die Proteinaddukte aus den Metaboliten des Antibiotikums.

Fazit: Nach den Daten dieser Studie erfolgt die allergische Reaktion nach Sulfamethoxazol-Exposition bei Patienten mit zystischer Fibrose nicht als direkte Antwort der T-Zellen auf das Antibiotikum selbst, sondern auf dessen durch antigenpräsentierende Zellen metabolisierte Produkte.

Andreas Fischer

Elsheikh $\mathrm{A}$ et al. Enhanced antigenicity leads to altered immunogenicity in sulfamethoxazole-hypersensititve patients with cystic fibrosis. J Allergy Clin Immunol 2011 Feb 26 [Epub ahead of print] 